\title{
MÉTODO EXTENDIDO DE HILDEBRAND EN LA PREDICCIÓN DE LA SOLUBILIDAD DEL KETOPROFENO EN MEZCLAS COSOLVENTES ETANOL + AGUA
}

\author{
Mauricio Gantiva y Fleming Martínez* \\ Departamento de Farmacia, Facultad de Ciencias, Universidad Nacional de Colombia, A.A. 14490, Bogotá D.C., Colombia
}

Recebido em 1/4/09; aceito em 24/7/09; publicado na web em 11/1/10

\begin{abstract}
EXTENDED HILDEBRAND SOLUBILITY APPROACH IN THE ESTIMATION OF KETOPROFEN SOLUBILITY IN ETHANOL + WATER COSOLVENT MIXTURES. Extended Hildebrand Solubility Approach (EHSA) developed by Martin et al. was applied to evaluate the solubility of ketoprofen (KTP) in ethanol + water cosolvent mixtures at $298.15 \mathrm{~K}$. Calculated values of molar volume and solubility parameter for KTP were used. A good predictive capacity of EHSA was found by using a regular polynomial model in order five to correlate the $W$ interaction parameter and the solubility parameters of cosolvent mixtures $\left(\delta_{\text {mix }}\right)$. Nevertheless, the deviations obtained in the estimated solubilities with respect to the experimental solubilities were on the same order like those obtained directly by means of an empiric regression of the logarithmic experimental solubilities as a function of $\delta_{\text {mix }}$ values.
\end{abstract}

Keywords: ketoprofen; extended Hildebrand solubility approach; cosolvent mixtures.

\section{INTRODUCCIÓN}

El ketoprofeno (KTP) es un derivado del ácido propiónico y farmacológicamente tiene acción analgésica y está clasificado como anti-inflamatorio no esteroidal (AINE). Por sus propiedades es ampliamente utilizado en la terapéutica actual para el tratamiento de diversos procesos agudos o crónicos relacionados con dolor e inflamación. ${ }^{1}$

De otro lado, es bien sabido que las formulaciones líquidas inyectables se caracterizan por suministrar una alta dosis de fármaco en un pequeño volumen de producto. Por esta razón, algunas propiedades fisicoquímicas tales como la solubilidad y los volúmenes ocupados por los principios activos y los otros componentes en la solución se tornan muy importantes para el diseñador farmacéutico, ya que el conocimiento de estas propiedades, así como el adecuado manejo y de ser posible, la predicción de estos fenómenos, facilita de manera sustancial el desarrollo de medicamentos líquidos. ${ }^{2}$

Por lo anteriormente expuesto, en la presente investigación se presenta un estudio fisicoquímico sobre la predicción de la solubilidad del KTP a 298,15 K en mezclas binarias formadas por etanol y agua. El estudio está basado en el Método Extendido de Solubilidad de Hildebrand (MESH) desarrollado para ser usado en sistemas de interés farmacéutico. ${ }^{3}$ Con este fin se determinó la solubilidad del KTP en los solventes puros y en diferentes mezclas cosolventes y se calculó el volumen molar y el parámetro de solubilidad del fármaco mediante algunos métodos basados en contribución de grupos; así mismo se utilizaron algunos datos de la literatura sobre las propiedades relativas a la fusión del fármaco. Esta investigación se constituye en una ampliación de lo presentado recientemente en la literatura sobre el estudio de la predicción de la solubilidad de otros AINEs en sistemas cosolventes utilizando el MESH. ${ }^{4}$

Es de anotar que en la literatura se han presentado otros métodos para la estimación de la solubilidad de fármacos en sistemas cosolventes, dentro de los cuales los más destacados son los desarrollados por Yalkowsky y Roseman, ${ }^{5}$ basado en la aditividad logarítmica de solubilidades, y por Jouyban y Acree, ${ }^{6}$ basado en la adición de un factor empírico al modelo original de Yalkowsky y Roseman, y que considera las desviaciones experimentales a la aditividad logarítmica. Una amplia revisión de todos estos métodos de estimación de solubilidad en mezclas cosolventes ha sido presentada recientemente por Jouyban. ${ }^{7}$

\footnotetext{
*e-mail: fmartinezr@unal.edu.co
}

\section{Aspectos teóricos}

La solubilidad ideal $\left(X_{2}^{\text {id }}\right)$ de un soluto sólido en un solvente líquido para obtener una solución líquida,${ }^{8}$ se calcula adecuadamente mediante la expresión:

$\log X_{2}^{i d}=-\frac{\Delta H_{\text {fus }}\left(T_{\text {fus }}-T\right)}{2,303 R T_{\text {fus }} T}$

en la cual, $\Delta H_{\text {fus }}$ es la entalpía de fusión del soluto, $R$ es la constante de los gases $\left(8,314 \mathrm{~J} \mathrm{~mol}^{-1} \mathrm{~K}^{-1}\right), T_{\text {fus }}$ es la temperatura absoluta de fusión del soluto y $T$ es la temperatura absoluta de la solución. Por otro lado, la solubilidad real $\left(X_{2}\right)$ se calcula adicionando el término de no-idealidad $\left(\log \gamma_{2}\right)$ a la expresión anterior, ${ }^{8}$ para obtener la ecuación:

$$
-\log X_{2}=\frac{\Delta H_{\text {fus }}\left(T_{\text {fus }}-T\right)}{2,303 R T_{\text {fus }} T}+\log \gamma_{2}
$$

El término $\gamma_{2}$ es el coeficiente de actividad del soluto en la solución saturada y debe ser determinado experimentalmente para soluciones reales, en las cuales se presentan interacciones de diversa índole, tales como los enlaces de hidrógeno, sin embargo se han desarrollado diferentes técnicas para hacer estimados razonables de este término. Un ejemplo específico de tales aproximaciones, lo constituyen las denominadas soluciones regulares introducidas por Hildebrand y Scatchard, en las cuales, a diferencia de las soluciones ideales, se permite un ligero cambio entálpico positivo, esto es, se requiere suministrar una pequeña cantidad de energía para que se conduzca el proceso de solución, mientras que el cambio entrópico es el correspondiente a una solución ideal. La solubilidad en las soluciones regulares ${ }^{9,10}$ se obtiene a partir de:

$$
-\log X_{2}=\frac{\Delta H_{\text {fus }}\left(T_{\text {fus }}-T\right)}{2,303 R T_{\text {fus }} T}+\frac{V_{2} \phi_{1}^{2}}{2,303 R T}\left(\delta_{1}-\delta_{2}\right)^{2}
$$

donde, $V_{2}$ es el volumen molar parcial del soluto $\left(\mathrm{cm}^{3} \mathrm{~mol}^{-1}\right), \phi_{1}$ es la fracción volumétrica del solvente en la solución saturada y $\delta_{1}$ y $\delta_{2}$ son los parámetros de solubilidad del solvente y del soluto, respectivamente. El parámetro de solubilidad se define como la raíz cuadrada de la densidad de energía cohesiva y se calcula como: $\delta=\left(\left(\Delta H_{v}-R T\right) / V_{l}\right)^{1 / 2}$, donde, $\Delta H_{v}$ es la entalpía de vaporización y $V_{l}$ es el volumen molar 
del líquido. En la literatura ${ }^{9-11}$ se encuentran disponibles los valores de parámetro de solubilidad para muchos solventes y también para algunos compuestos sólidos, entre ellos varios fármacos.

La mayoría de soluciones de interés farmacéutico se desvían notoriamente del comportamiento de las soluciones regulares (debido al tipo de interacciones presentes, en particular la formación de enlaces de hidrógeno, y a la diferencia entre los volúmenes molares de solutos y solventes). Así, a comienzos de los años 80 del siglo pasado, Martin et al. plantearon el $\mathrm{MESH},{ }^{3}$ el cual ha resultado muy útil para estimar la solubilidad de varios fármacos en sistemas cosolventes binarios y ternarios.,

Si se introduce el término volumétrico-energético, $A$, definido como $V_{2} \phi_{1}^{2} /(2,303 R T)$, la solubilidad real de un soluto en cualquier sistema solvente se puede calcular a partir de:

$-\log X_{2}=-\log X_{2}^{\mathrm{id}}+A\left(\delta_{1}^{2}+\delta_{2}^{2}-2 W\right)$

en la cual, el término $W$ es igual a $2 K \delta_{1} \delta_{2}$, y en donde $K$ es el parámetro de Walker, introducido inicialmente para el estudio de soluciones de polímeros. ${ }^{9} \mathrm{El}$ factor $W$ compensa las desviaciones respecto al comportamiento de soluciones regulares, y se calcula a partir de datos experimentales como:

$W=0,5 \times\left(\delta_{1}^{2}+\delta_{2}^{2}-\frac{\log \gamma_{2}}{A}\right)$

donde, $\gamma_{2}$ como se indicó anteriormente, es el coeficiente de actividad del soluto en la solución y se calcula como el cociente $X_{2}^{i d} / X_{2}$.

Los valores obtenidos experimentalmente para el factor $W$ pueden ser correlacionados mediante análisis de regresión usando polinomios regulares de orden superior en función del parámetro de solubilidad de las mezclas solventes así:

$W=C_{0}+C_{1} \delta_{1}+C_{2} \delta_{1}^{2}+C_{3} \delta_{1}^{3} \ldots . .+C_{n} \delta_{1}^{n}$

Estos modelos polinómicos pueden ser utilizados para estimar la solubilidad del fármaco por cálculo reverso (regresión en el cálculo, usando la ecuación obtenida a partir de los datos experimentales), resolviendo esta propiedad a partir del factor $W$ obtenido de la regresión polinómica.

\section{PARTE EXPERIMENTAL}

\section{Materiales}

Se utilizaron los siguientes materiales: ketoprofeno USP; ${ }^{12}$ etanol absoluto R.A. Merck (EtOH); agua destilada (W) conductividad <2 $\mu \mathrm{S} \mathrm{cm}^{-1}$; tamiz molecular Merck (números 3 y 4); filtros Durapore ${ }^{\circledR}$ 0,45 $\mu \mathrm{m}$ Millipore Corp.; material aforado de vidrio.

\section{Preparación de las mezclas cosolventes}

Todas las mezclas etanol + agua se prepararon por pesada en cantidades cercanas a 40,00 g en una balanza analítica Ohaus Pioneer TM PA214 (sensibilidad $\pm 0.1 \mathrm{mg}$ ), en concentraciones desde 10 hasta $90 \% \mathrm{~m} / \mathrm{m}$ variando en $10 \% \mathrm{~m} / \mathrm{m}$, con lo cual se estudiaron nueve mezclas binarias y los dos solventes puros.

\section{Determinación de la solubilidad}

En frascos ámbar con tapa rosca se adicionó un exceso de KTP a $20 \mathrm{~cm}^{3}$ de cada mezcla cosolvente. Las mezclas sólido-líquido se colocaron en un baño de ultrasonido (Elmasonic E $60 \mathrm{H}$ ) durante 15 min y posteriormente se colocaron en un baño termostático con agitación (Julabo SW23) ajustado a 313,15 \pm 0,05 K durante al menos 5 días hasta alcanzar la saturación. Este tiempo se estableció determinando la concentración del fármaco hasta obtener valor constante. Posteriormente la temperatura se disminuyó a 298,15 \0,05 K durante al menos 2 días, con el fin de permitir la precipitación del exceso de fármaco. Transcurrido este tiempo se filtraron las soluciones saturadas a temperatura constante para remover partículas no disueltas antes de tomar alícuotas. Después de realizar las diluciones requeridas se determinaron las concentraciones de fármaco mediante espectrofotometría UV (UV/VIS BioMate 3 Thermo Electron Company spectrophotometer). Para permitir la conversión de expresiones de concentración se determinó la densidad de las soluciones saturadas utilizando un densímetro digital (DMA 45 Anton Paar) conectado a un baño termostático de recirculación (Neslab RTE 10 Digital One Thermo Electron Company). Todos los procedimientos fueron realizados por triplicado.

\section{RESULTADOS Y DISCUSIÓN}

En la Tabla 1 se presentan algunas propiedades fisicoquímicas del fármaco en estudio tomadas de la literatura, ${ }^{13,14}$ mientras que en la Tabla 2 se presenta la composición másica y volumétrica de las mezclas solventes EtOH $+\mathrm{W}$ junto con los respectivos parámetros de solubilidad a 298,15 K. ${ }^{15}$ La fracción volumétrica $(f)$ se halló a partir de los volúmenes molares parciales de etanol y agua y de la composición cosolvente expresada en fracción molar. ${ }^{15} \mathrm{El}$ parámetro de solubilidad de las mezclas cosolventes $\left(\delta_{\text {mix }}\right)$ se calculó mediante aditividad en términos volumétricos de acuerdo con: $\delta_{\text {mix }}=\sum_{i=1}^{n} \delta_{i} f_{i}$, donde $\delta_{i}$ es el parámetro de solubilidad de cada componente, en este caso $26,59 \mathrm{MPa}^{1 / 2}$ para el EtOH y 47,86 $\mathrm{MPa}^{1 / 2}$ para el W, a 298,15 K. ${ }^{16}$

Tabla 1. Propiedades fisicoquímicas del KTP

\begin{tabular}{|c|c|c|c|}
\hline Estructura molecular (a) & $\begin{array}{c}\text { Masa molar } \\
\mathrm{g} \mathrm{mol}^{-1} \text { (a) }\end{array}$ & $\begin{array}{l}\text { Punto de fusión } \\
\qquad \mathrm{K}^{(\mathrm{b})}\end{array}$ & $\begin{array}{c}\Delta H_{\text {fus }} \\
\mathrm{kJ} \mathrm{mol}^{-1} \text { (b) }\end{array}$ \\
\hline $\mathrm{COOH}$ & 254,28 & 367,1 & 21,0 \\
\hline
\end{tabular}

(a) Budavari et al. ${ }^{13}$; (b) Perlovich et al. ${ }^{14}$

Así mismo, en la Tabla 2 se presenta la solubilidad del fármaco, expresada en molaridad y fracción molar, observándose que la solubilidad experimental varía de forma no lineal con la composición cosolvente. Todos los valores de solubilidad del KTP fueron mayores que los reportados para el naproxeno en las mismas mezclas cosolventes, ${ }^{17}$ mientras que los mismos valores fueron menores que los reportados para ibuprofeno en las mezclas donde se obtuvo una sola fase líquida. ${ }^{18}$ De forma similar, el naproxeno y el ibuprofeno también son AINES derivados del ácido propiónico. En la Figura 1 se presenta la solubilidad experimental y la solubilidad calculada usando el modelo de soluciones regulares (Ecuación 3) en función del parámetro de solubilidad de las mezclas disolventes. Puede observarse que la solubilidad experimental del KTP en todos los casos es mayor que la estimada por el método de Hildebrand-Scatchard, lo cual es similar a lo observado en el estudio del naproxeno en este mismo sistema cosolvente. ${ }^{4}$ Sin embargo, la solubilidad experimental del naproxeno en etanol puro es menor que la estimada mediante el modelo de soluciones regulares. ${ }^{4}$ Este resultado podría ser una consecuencia de la menor solubilidad del naproxeno en relación con el KTP.

Para este tipo de tratamientos predictivos, tradicionalmente se ha asumido como constante el valor del volumen molar del soluto en la solución. ${ }^{9,10}$ Propiedad que en el caso de compuestos sólidos 
Tabla 2. Composición de las mezclas solventes en masa y volumen, parámetros de solubilidad de la mezclas cosolventes, solubilidad del KTP, fracción volumétrica del solvente y coeficiente de actividad del KTP a 298,15 K

\begin{tabular}{|c|c|c|c|c|c|c|}
\hline \multirow{2}{*}{$\mathrm{EtOH} / \% \mathrm{~m} / \mathrm{m}$} & \multirow{2}{*}{$f \mathrm{EtOH}$} & \multirow{2}{*}{$\delta_{1} / \mathrm{MPa}^{1 / 2(\mathrm{a})}$} & \multicolumn{2}{|c|}{ Solubilidad } & \multirow[b]{2}{*}{$\phi_{1}$} & \multirow[b]{2}{*}{$\log \gamma$} \\
\hline & & & $\mathrm{Mol} \mathrm{L}^{-1}$ & $X_{2}$ & & \\
\hline 0,00 & 0,0000 & 47,86 & $4,29 \times 10^{-4}$ & $7,73 \times 10^{-6}$ & 0,9999 & 4,421 \\
\hline 10,00 & 0,1140 & 45,44 & $6,60 \times 10^{-4}$ & $1,28 \times 10^{-5}$ & 0,9999 & 4,201 \\
\hline 20,00 & 0,2275 & 43,02 & $1,34 \times 10^{-3}$ & $2,81 \times 10^{-5}$ & 0,9997 & 3,861 \\
\hline 30,00 & 0,3401 & 40,63 & $4,39 \times 10^{-3}$ & $1,01 \times 10^{-4}$ & 0,9991 & 3,305 \\
\hline 40,00 & 0,4505 & 38,28 & $1,43 \times 10^{-2}$ & $3,59 \times 10^{-4}$ & 0,9973 & 2,754 \\
\hline 50,00 & 0,5580 & 36,00 & $4,77 \times 10^{-2}$ & $1,32 \times 10^{-3}$ & 0,9910 & 2,189 \\
\hline 60,00 & 0,6614 & 33,80 & 0,133 & $4,18 \times 10^{-3}$ & 0,9750 & 1,688 \\
\hline 70,00 & 0,7594 & 31,71 & 0,263 & $9,58 \times 10^{-3}$ & 0,9505 & 1,328 \\
\hline 80,00 & 0,8499 & 29,79 & 0,468 & $2,06 \times 10^{-2}$ & 0,9105 & 0,996 \\
\hline 90,00 & 0,9302 & 28,08 & 0,618 & $3,33 \times 10^{-2}$ & 0,8792 & 0,787 \\
\hline 100,00 & 1,0000 & 26,59 & 0,672 & $4,41 \times 10^{-2}$ & 0,8667 & 0,665 \\
\hline
\end{tabular}

(a) En el caso de las mezclas cosolventes, el valor $\delta_{1}$ fue calculado como $\delta_{\text {mix }}=\sum_{i=1}^{n} \delta_{i} f_{i}$

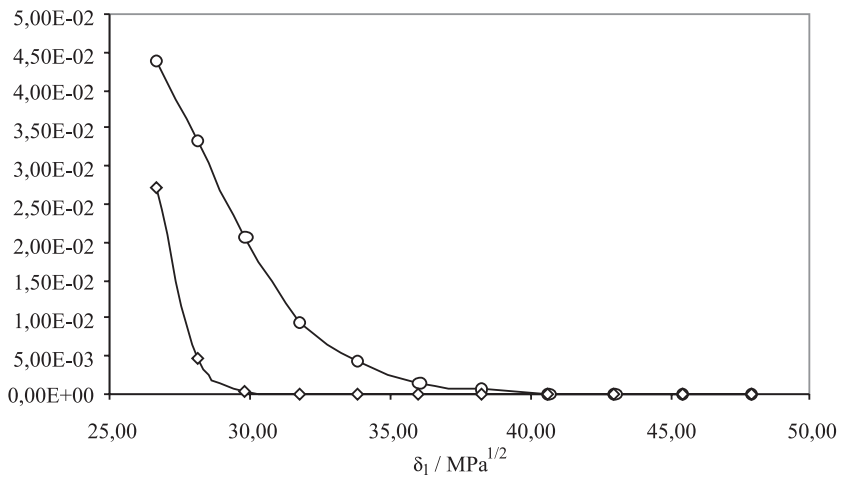

Figura 1. Solubilidad experimental $(\bigcirc)$ y solubilidad calculada según el modelo de soluciones regulares de Hildebrand $(\diamond)$ del KTP en función del parámetro de solubilidad de las mezclas cosolventes a 298,15 K

(a causa de la gran dificultad en su determinación experimental), es calculado por métodos de contribución de grupos, tales como el propuesto por Fedors. ${ }^{19}$ Por lo tanto, en este trabajo se utilizó el valor calculado por este método $\left(195,6 \mathrm{~cm}^{3} \mathrm{~mol}^{-1}\right.$, Tabla 3$)$. De otro lado, la fracción volumétrica del solvente en la solución saturada se calculó según la expresión:

$\phi_{1}=\frac{V_{1}\left(1-X_{2}\right)}{V_{1}\left(1-X_{2}\right)+V_{2} X_{2}}$

en la cual, el término $V_{1}$ es el volumen molar de la mezcla cosolvente. Los valores experimentales de $V_{1}$ fueron tomados de Jiménez et al. ${ }^{15}$ De otro lado, si bien es cierto que en investigaciones similares, ${ }^{4,9,10} \mathrm{el}$ término $V_{1}$ ha sido calculado mediante el método de aditividad lineal de volúmenes como: $V_{1-\operatorname{mix}}=\sum_{i=1}^{n} V_{1 i} f_{i}$, es de resaltar que los volúmenes de mezcla no son propiamente aditivos en el caso de mezclas cosolventes con fuerte presencia de enlaces de hidrógeno y grandes diferencias en los volúmenes molares de los componentes. Por esta razón, en esta investigación se decidió utilizar los valores presentados en la literatura, ${ }^{15}$ en lugar de los calculados considerando aditividad de volúmenes. Los valores de $\phi_{1}$ también se presentan en la Tabla 2, así como lo valores de $\gamma_{2}$ (como logaritmo decimal) calculados utilizando la solubilidad ideal del fármaco (0,2039 a 298,15 K).
De otro lado, en la Tabla 4 se presentan los factores $A, K$ y $W$. Para el cálculo del parámetro $W$ se utilizó como valor del parámetro de solubilidad del fármaco, el obtenido mediante el método de van Krevelen descrito por Barton $\left(22,46 \mathrm{MPa}^{1 / 2}\right.$, Tabla 3$) .{ }^{16}$ Esto se hizo así ya que en este sistema no se obtiene el máximo de solubilidad en alguna mezcla cosolvente sino que este valor se obtiene en EtOH puro.

Como se indicó previamente, el factor $W$ considera las desviaciones presentadas por las soluciones reales respecto a las soluciones regulares, debidas principalmente a interacciones específicas de tipo unión de hidrógeno. El fármaco y los dos solventes considerados en este estudio pueden fácilmente establecer estas uniones, ya sea como donores o como aceptores de hidrógeno, gracias a los grupos hidroxilo de los dos solventes, e hidroxilo y carbonilo del fármaco (Tabla 1).

En la Figura 2 se presenta la variación del factor $W$ con el parámetro de solubilidad de las mezclas cosolventes, donde puede observarse un comportamiento que se desvía de la linealidad. En la Tabla 4 se presentan los valores calculados o normalizados del factor $W$, obtenidos utilizando modelos de grados 1 a 5, donde es bien claro que estos valores dependen del tipo de modelo polinómico regular utilizado en la regresión. Situaciones similares han sido descritas en la literatura al comparar los resultados obtenidos con polinomios de segundo y de cuarto orden., ${ }^{3,10}$

En la Tabla 5 se presentan los valores de solubilidad obtenidos por cálculo reverso utilizando los diferentes valores del factor $W$ calculados mediante las regresiones polinómicas de diferente orden que han sido presentados en la Tabla 4. Como criterio de comparación, en la Tabla 5 también se presentan los porcentajes de diferencia entre la solubilidad experimental y la calculada usando los diferentes modelos de regresión.

Puede observarse que en la medida en que los polinomios se van haciendo más complejos, se van obteniendo mejores concordancias entre los valores calculados y los experimentales, lo que se confirma al verificar los porcentajes de desviación promedios (2,5\% para orden 5). De manera similar a lo encontrado en otros estudios, ${ }^{3,4}$ en este caso el incremento más notable en la concordancia obtenida se presenta al pasar del orden 1 al 2, si bien en los siguientes pasos también se encuentran incrementos visibles, en particular el paso del orden 2 al 3. Es de resaltar que el valor de solubilidad obtenido en la mezcla con $\delta_{1}$ $=36,00 \mathrm{MPa}^{1 / 2}$, usando el modelo de primer orden, es completamente absurdo puesto que es igual a 1,15, expresado en fracción molar. Este resultado anómalo sería consecuencia de la alta desviación numérica 
Tabla 3. Aplicación de los métodos de Fedors y van Krevelen para la estimación del volumen molar y los parámetros de solubilidad parciales de Hansen y total de Hildebrand para el KTP

\begin{tabular}{|c|c|c|c|c|c|}
\hline \multirow{2}{*}{ Grupo o átomo } & \multirow{2}{*}{ Cantidad } & \multicolumn{2}{|l|}{ Fedors ${ }^{(a)}$} & \multicolumn{2}{|l|}{ Van Krevelen $^{(b)}$} \\
\hline & & $V / \mathrm{cm}^{3} \mathrm{~mol}^{-1}$ & $F_{d} / \mathrm{J}^{1 / 2} \mathrm{~cm}^{3 / 2} \mathrm{~mol}^{-1}$ & $F_{p}^{2} / \mathrm{J} \mathrm{cm}^{3} \mathrm{~mol}^{-2}$ & $U_{h} / \mathrm{J} \mathrm{mol}^{-1}$ \\
\hline$-\mathrm{COOH}$ & 1 & 28,5 & 530 & $420^{2}$ & 10000 \\
\hline$>\mathrm{C}=\mathrm{O}$ & 1 & 10,8 & 290 & $770^{2}$ & 2000 \\
\hline$-\mathrm{CH}_{3}$ & 1 & 33,5 & 420 & $0^{2}$ & 0 \\
\hline$>\mathrm{CH}-$ & 1 & $-1,0$ & 80 & $0^{2}$ & 0 \\
\hline Fenilo & 1 & 71,4 & 1430 & $110^{2}$ & 0 \\
\hline \multirow[t]{4}{*}{ Fenileno } & 1 & 52,4 & 1270 & $110^{2}$ & 0 \\
\hline & & 195,6 & 4020 & 793500 & 12000 \\
\hline & & & $\begin{array}{l}\delta_{d}=(4020 / 195,6) \\
=20,55 \mathrm{MPa}^{1 / 2(\mathrm{c})}\end{array}$ & $\begin{aligned} \delta_{p}= & \left((793500)^{1 / 2} / 195,6\right) \\
= & 4,55 \mathrm{MPa}^{1 / 2(\mathrm{~d})}\end{aligned}$ & $\begin{aligned} \delta_{h} & =(12000 / 195,6)^{1 / 2} \\
& =7,83 \mathrm{MPa}^{1 / 2(\mathrm{e})}\end{aligned}$ \\
\hline & & & \multicolumn{3}{|c|}{$\delta_{T}=\left(20,55^{2}+4,55^{2}+7,83^{2}\right)^{1 / 2}=22,46 \mathrm{MPa}^{1 / 2(\mathrm{f})}$} \\
\hline
\end{tabular}

(a) Calculados de acuerdo a los valores presentados por Fedors ${ }^{19}$; (b) calculados de acuerdo a los valores y procedimientos descritos por Barton ${ }^{16}$; ${ }^{\text {(c) }}$ parámetro parcial por fuerzas de dispersión; ${ }^{(\mathrm{d})}$ parámetro parcial por fuerzas dipolares; ${ }^{(e)}$ parámetro parcial por enlaces de hidrógeno; ${ }^{(\mathrm{f})}$ parámetro de solubilidad total.

Tabla 4. Factor $A$, factor $K$, factor $W$ experimental y calculado utilizando modelos polinómicos regulares de órdenes 1,2 , 3,4 y 5 a 298,15 K

\begin{tabular}{|c|c|c|c|c|c|c|c|c|}
\hline$\delta_{1} / \mathrm{MPa}^{1 / 2}$ & $10^{2} A / \mathrm{cm}^{3} \mathrm{~J}^{-1}$ & $K / \mathrm{J} \mathrm{cm}^{-3}$ & $W_{\text {expt }} / \mathrm{J} \mathrm{cm}^{-3}$ & 1 & 2 & $\begin{array}{c}\text { Iculado } / \mathrm{J} \mathrm{cm} \\
3\end{array}$ & 4 & 5 \\
\hline 47,86 & 6,8073 & 0,63498 & 1365,266 & 1329,333 & 1363,728 & 1365,095 & 1365,355 & 1365,297 \\
\hline 45,44 & 6,8067 & 0,61424 & 1253,758 & 1242,784 & 1254,289 & 1253,815 & 1253,490 & 1253,625 \\
\hline 43,02 & 6,8049 & 0,59473 & 1149,420 & 1156,579 & 1151,018 & 1149,871 & 1149,628 & 1149,615 \\
\hline 40,63 & 6,7969 & 0,57713 & 1053,313 & 1071,103 & 1054,272 & 1053,268 & 1053,315 & 1053,210 \\
\hline 38,28 & 6,7712 & 0,56095 & 964,614 & 987,266 & 964,845 & 964,446 & 964,703 & 964,645 \\
\hline 36,00 & 6,6865 & 0,54653 & 883,685 & 905,669 & 883,005 & 883,339 & 883,598 & 883,647 \\
\hline 33,80 & 6,4721 & 0,53373 & 810,247 & 827,149 & 809,092 & 809,996 & 810,070 & 810,171 \\
\hline 31,71 & 6,1516 & 0,52246 & 744,207 & 752,730 & 743,421 & 744,507 & 744,337 & 744,379 \\
\hline 29,79 & 5,6444 & 0,51346 & 686,997 & 684,025 & 686,579 & 687,332 & 687,035 & 686,963 \\
\hline 28,08 & 5,2624 & 0,50658 & 638,886 & 623,013 & 639,148 & 639,061 & 638,900 & 638,797 \\
\hline 26,59 & 5,1142 & 0,50170 & 599,278 & 570,018 & 600,276 & 598,945 & 599,243 & 599,324 \\
\hline
\end{tabular}

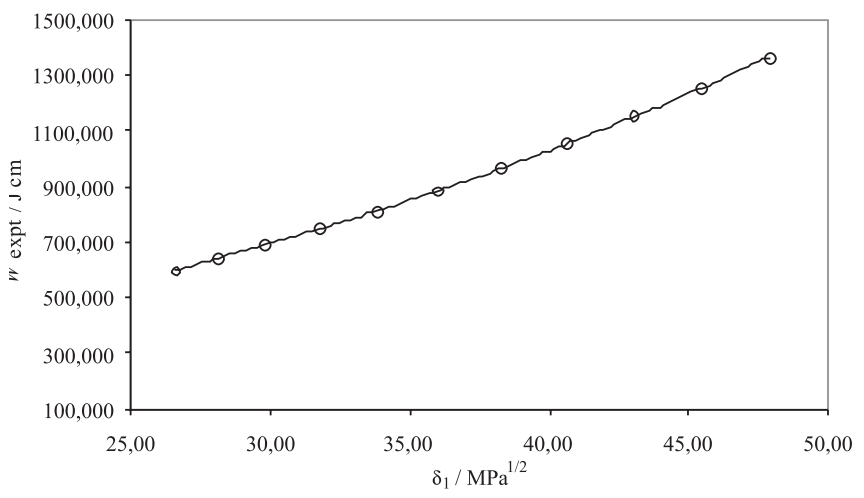

Figura 2. Variación del factor $W$ en función del parámetro de solubilidad de las mezclas cosolventes a $298,15 \mathrm{~K}$

presentada por las mezclas de composición intermedia (la desviación en este punto es del $86971 \%$ ) al usar el modelo lineal. Por lo tanto, para los cálculos siguientes se utilizó el modelo con menor desviación general (orden 5, Ecuación 8).

$W=C_{0}+C_{1} \delta_{1}+C_{2} \delta_{1}^{2}+C_{3} \delta_{1}^{3}+C_{4} \delta_{1}^{4}+C_{5} \delta_{1}^{5}$ en la cual, $C_{0}=+1202,305, C_{1}=-133,0775, C_{2}=+7,782223, C_{3}$ $=-0,1942609, C_{4}=+2,502179 \times 10^{-3}, C_{5}=-1,330775 \times 10^{-5}, \mathrm{y} r^{2}$ $=0,9999998$.

Si bien la desviación general en la Ecuación 8 es de 2,5\%, los valores más altos se encuentran en las mezclas cosolventes conteniendo alrededor del 20 y $70 \% \mathrm{~m} / \mathrm{m}$ de EtOH. Es de anotar que en la mezcla del $20 \%$ de EtOH aún puede presentarse agua voluminosa estructurada por enlaces de hidrógeno. Para evaluar este posible efecto, en la Figura 3 se presenta el logaritmo decimal de la solubilidad en función de la polaridad de las mezclas. Así mismo se presenta la línea de solubilidad aditiva logarítmica según lo esperado de acuerdo al tratamiento de Yalkowsky y Valvani. ${ }^{20}$ Según esta figura se tiene que en las mezclas más polares la solubilidad es menor que la esperada según el modelo log-lineal y que en la mezcla cercana al $40 \% \mathrm{~m} / \mathrm{m}$ de $\operatorname{EtOH}\left(38,1 \mathrm{MPa}^{1 / 2}\right)$ se pasa de desviaciones negativas a positivas.

Una explicación posible para las desviaciones negativas presentadas a bajas proporciones de cosolvente, podría hallarse en el trabajo de Kimura et al. ${ }^{21}$ quienes obtuvieron comportamientos similares en los calores de disolución de 1-metil-2-pirrolidinona en mezclas alcoholagua. Según estos resultados, a bajas concentraciones de cosolvente, el agua retiene su habilidad para formar estructuras ordenadas.

Como es bien sabido, los cosolventes poseen grupos polares y no polares en su estructura molecular y por lo tanto, se espera que la hidra- 
Tabla 5. Solubilidad calculada utilizando factor $W$ obtenido por modelos de regresión de órdenes 1, 2, 3, 4 y 5, y porcentaje de diferencia respecto al valor experimental a $298,15 \mathrm{~K}$

\begin{tabular}{|c|c|c|c|c|c|c|c|c|c|c|}
\hline \multirow{2}{*}{$\delta_{1} / \mathrm{MPa}^{1 / 2}$} & \multicolumn{5}{|c|}{$X_{2}$ calculado } & \multicolumn{5}{|c|}{$\%$ desv. ${ }^{(a)}$} \\
\hline & 1 & 2 & 3 & 4 & 5 & 1 & 2 & 3 & 4 & 5 \\
\hline 47,86 & $9,91 \times 10^{-11}$ & $4,77 \times 10^{-6}$ & $7,33 \times 10^{-6}$ & $7,95 \times 10^{-6}$ & $7,80 \times 10^{-6}$ & 100 & 38,3 & 5,2 & 2,8 & 1,0 \\
\hline 45,44 & $4,11 \times 10^{-7}$ & $1,52 \times 10^{-5}$ & $1,31 \times 10^{-5}$ & $1,18 \times 10^{-5}$ & $1,23 \times 10^{-5}$ & 97 & 18,1 & 1,8 & 8,1 & 4,1 \\
\hline 43,02 & $2,65 \times 10^{-4}$ & $4,63 \times 10^{-5}$ & $3,23 \times 10^{-5}$ & $3,00 \times 10^{-5}$ & $2,98 \times 10^{-5}$ & 842 & 65,0 & 15,2 & 6,7 & 6,3 \\
\hline 40,63 & $2,65 \times 10^{-2}$ & $1,36 \times 10^{-4}$ & $9,96 \times 10^{-5}$ & $1,01 \times 10^{-4}$ & $9,78 \times 10^{-5}$ & 26103 & 35,0 & 1,4 & 0,1 & 3,2 \\
\hline 38,28 & 0,419 & $3,86 \times 10^{-4}$ & $3,41 \times 10^{-4}$ & $3,69 \times 10^{-4}$ & $3,62 \times 10^{-4}$ & 116743 & 7,4 & 5,1 & 2,8 & 0,9 \\
\hline 36,00 & 1,15 & $1,07 \times 10^{-3}$ & $1,19 \times 10^{-3}$ & $1,28 \times 10^{-3}$ & $1,30 \times 10^{-3}$ & 86971 & 18,9 & 10,1 & 2,7 & 1,2 \\
\hline 33,80 & 0,644 & $2,96 \times 10^{-3}$ & $3,88 \times 10^{-3}$ & $3,97 \times 10^{-3}$ & $4,09 \times 10^{-3}$ & 15313 & 29,1 & 7,2 & 5,1 & 2,2 \\
\hline 31,71 & 0,107 & $7,67 \times 10^{-3}$ & $1,04 \times 10^{-2}$ & $9,94 \times 10^{-3}$ & $1,01 \times 10^{-2}$ & 1019 & 20,0 & 8,9 & 3,8 & 5,0 \\
\hline 29,79 & $9,52 \times 10^{-3}$ & $1,85 \times 10^{-2}$ & $2,25 \times 10^{-2}$ & $2,08 \times 10^{-2}$ & $2,04 \times 10^{-2}$ & 54 & 10,3 & 9,1 & 1,0 & 0,9 \\
\hline 28,08 & $7,11 \times 10^{-4}$ & $3,55 \times 10^{-2}$ & $3,47 \times 10^{-2}$ & $3,34 \times 10^{-2}$ & $3,26 \times 10^{-2}$ & 98 & 6,6 & 4,3 & 0,3 & 2,1 \\
\hline \multirow[t]{4}{*}{26,59} & $4,48 \times 10^{-5}$ & $5,58 \times 10^{-2}$ & $4,08 \times 10^{-2}$ & $4,37 \times 10^{-2}$ & $4,46 \times 10^{-2}$ & 100 & 26,5 & 7,5 & 0,8 & 1,1 \\
\hline & & & & \multicolumn{2}{|c|}{ Promedio ${ }^{(b)}$} & 22495 & 25 & 6,9 & 3,1 & 2,5 \\
\hline & & & & \multicolumn{2}{|c|}{ Error típico ${ }^{(b)}$} & 12264 & 5 & 1,2 & 0,8 & 0,6 \\
\hline & & & & \multicolumn{2}{|c|}{ Desviación estándar (b) } & 40675 & 17 & 4,0 & 2,6 & 1,9 \\
\hline
\end{tabular}

${ }^{(a)}$ Calculado como $100 \mathrm{x} \mid X_{2}$ expt $-X_{2}$ calc $\mid / X_{2}$ expt; (b) calculado considerando los valores obtenidos en los dos solventes puros y en las nueve mezclas binarias.

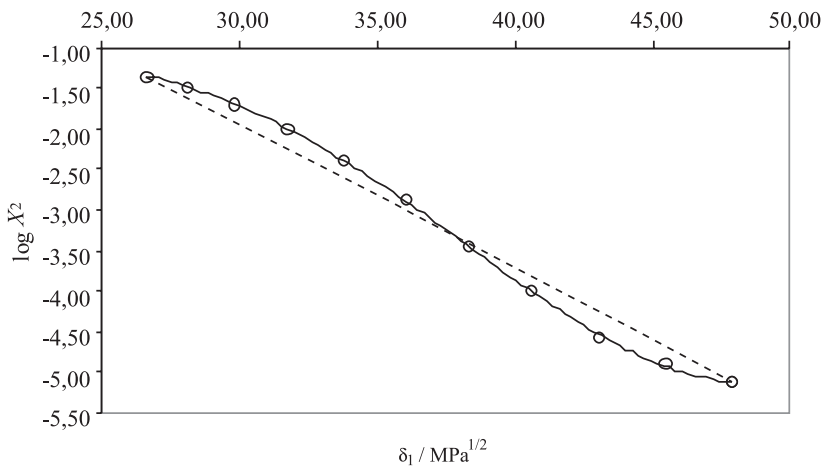

Figura 3. Logaritmo decimal de la solubilidad experimental (—) y comportamiento aditivo logarítmico (------) de la solubilidad del KTP en función del parámetro de solubilidad de las mezclas cosolventes a 298,15 K

tación hidrofóbica ocurra alrededor de las partes no polares del cosolvente (estructuración del agua formando agregados del tipo “témpano"), mientras que los enlaces de hidrógeno se forman entre los grupos polares y el agua. Aunque los alcoholes de pequeña masa molar generalmente han sido considerados como compuestos polares, Matsumoto et al..$^{22}$ han presentado evidencia de la influencia del grupo metilo terminal de estos cosolventes sobre la estructuración del agua, basados en el estudio de entalpías molares de exceso. Estas interacciones entre los alcoholes y el agua podrían reducir la interacción entre el agua y el KTP, llevando a una solubilidad inferior a la esperada según la línea de aditividad.

En cambio, a altas concentraciones de cosolvente, la estructura tridimensional del agua se pierde y por lo tanto, las moléculas de agua estarían más disponibles para interactuar con las moléculas del fármaco, lo cual a su vez, llevaría a obtener solubilidades relativamente altas. Si bien, es necesario indicar que la descripción anterior no explicaría la mayor solubilidad de este fármaco en el etanol puro. Otra explicación plausible para las altas desviaciones encontradas estaría dada en función de un posible fenómeno de auto-asociación del fármaco en la solución saturada, ${ }^{23}$ sin embargo para verificar esto en el presente caso se requeriría de otro tipo de evidencia experimental, tal como el estudio de los coeficientes de distribución del KTP en función de la concentración, en diferentes sistemas solvente orgánico/agua.

En la literatura ${ }^{17}$ se ha presentado evidencia de tipo termodinámico para explicar el comportamiento del ibuprofeno en este mismo sistema cosolvente, en términos del cambio de conducción para el proceso de solución de ese fármaco, al pasar de una conducción entrópica por debajo del $30 \%$ de $\mathrm{EtOH}$ a una conducción entálpica en concentraciones mayores del cosolvente. Siendo explicados estos resultados como una consecuencia de la pérdida de estructura del agua presente alrededor de los grupos no polares de ese fármaco, la cual se encontraba inicialmente asociada entre sí por hidratación hidrofóbica. Esta desestructuración se produce en la medida en que se va reemplazando el agua por etanol.

Una reflexión que podría surgir respecto a la concordancia obtenida al comparar la solubilidad experimental con la calculada a partir de modelos de regresión construidos sobre datos experimentales directos o procesados, es la relativa a la justificación de cálculos complejos involucrando otras variables del sistema (Ecuación 4 y Tablas 5 y 6), en lugar de la simple regresión de solubilidad en función del parámetro de solubilidad de las mezclas cosolventes (Tabla 1, Figuras 1 y 3 ). Por lo tanto, en la Tabla 6 se comparan las solubilidades experimentales, las calculadas directamente como logaritmo decimal de $X_{2}$ mediante regresión polinómica regular de cuarto grado (Figura 3, Ecuación 9), y las calculadas indirectamente involucrando los parámetros $W$ obtenidos del polinomio de quinto grado (Tabla 5, Ecuación 8). Así mismo, se presentan en esta tabla los respectivos porcentajes de diferencia respecto al valor experimental.

$$
\log X_{2}=C_{0}+C_{1} \delta_{1}+C_{2} \delta_{1}^{2}+C_{3} \delta_{1}^{3}+C_{4} \delta_{1}^{4}
$$

en la cual, $C_{0}=+0,6399202, C_{1}=-0,6405075, C_{2}=+5,251898 \times 10^{-2}$, $C_{3}=-1,556784 \times 10^{-3}, C_{4}=+1,434751 \times 10^{-5}$, y $r^{2}=0,9998570$.

En la Tabla 6 puede observarse que aparentemente no hay diferencias significativas entre los valores calculados por los dos métodos, lo que se verifica mediante los promedios de desviación, para los cálculos directo (3,0\%) y por el Método Extendido de Hildebrand (2,5\%), respectivamente. Los resultados previos estarían demostrando una 
Tabla 6. Comparación de los valores de solubilidad calculados directamente y mediante el método extendido de Hildebrand

\begin{tabular}{|c|c|c|c|c|c|}
\hline \multirow{2}{*}{$\delta_{1} / \mathrm{MPa}^{1 / 2}$} & \multicolumn{3}{|c|}{$X_{2}$} & \multicolumn{2}{|c|}{$\%$ desv. (a) } \\
\hline & Expt & Calc. direct. (b) & Calc. $W^{(\mathrm{c})}$ & Calc. direct. & Calc. $W$ \\
\hline 47,86 & $7,73 \times 10^{-6}$ & $7,87 \times 10^{-6}$ & $7,80 \times 10^{-6}$ & 1,8 & 1,0 \\
\hline 45,44 & $1,28 \times 10^{-5}$ & $1,20 \times 10^{-5}$ & $1,23 \times 10^{-5}$ & 6,1 & 4,1 \\
\hline 43,02 & $2,81 \times 10^{-5}$ & $3,00 \times 10^{-5}$ & $2,98 \times 10^{-5}$ & 6,8 & 6,3 \\
\hline 40,63 & $1,01 \times 10^{-4}$ & $9,92 \times 10^{-5}$ & $9,78 \times 10^{-5}$ & 1,7 & 3,2 \\
\hline 38,28 & $3,59 \times 10^{-4}$ & $3,65 \times 10^{-4}$ & $3,62 \times 10^{-4}$ & 1,6 & 0,9 \\
\hline 36,00 & $1,32 \times 10^{-3}$ & $1,30 \times 10^{-3}$ & $1,30 \times 10^{-3}$ & 1,9 & 1,2 \\
\hline 33,80 & $4,18 \times 10^{-3}$ & $4,01 \times 10^{-3}$ & $4,09 \times 10^{-3}$ & 4,1 & 2,2 \\
\hline 31,71 & $9,58 \times 10^{-3}$ & $1,02 \times 10^{-2}$ & $1,01 \times 10^{-2}$ & 6,0 & 5,0 \\
\hline 29,79 & $2,06 \times 10^{-2}$ & $2,04 \times 10^{-2}$ & $2,04 \times 10^{-2}$ & 0,8 & 0,9 \\
\hline 28,08 & $3,33 \times 10^{-2}$ & $3,29 \times 10^{-2}$ & $3,26 \times 10^{-2}$ & 1,2 & 2,1 \\
\hline \multirow[t]{4}{*}{26,59} & $4,41 \times 10^{-2}$ & $4,43 \times 10^{-2}$ & $4,46 \times 10^{-2}$ & 0,3 & 1,1 \\
\hline & \multicolumn{3}{|c|}{ Promedio $^{(\mathrm{d})}$} & 3,0 & 2,5 \\
\hline & \multicolumn{3}{|c|}{ Error típico ${ }^{(\mathrm{d})}$} & 0,7 & 0,6 \\
\hline & \multicolumn{3}{|c|}{ Desviación estándar ${ }^{(d)}$} & 2,4 & 1,9 \\
\hline
\end{tabular}

${ }^{\text {(a) }}$ Calculado como $100 \mathrm{x} \mid X_{2}$ expt $-X_{2}$ calc $\mid / X_{2}$ expt; ${ }^{\text {(b) }}$ calculado usando la Ec. 9; ${ }^{\text {(c) }}$ calculado usando la Ec. 8; (d) calculado considerando los valores obtenidos en los dos solventes puros y en las nueve mezclas binarias.

escasa practicidad del MESH, método que adicionalmente implica la determinación de algunas propiedades relativas a la fusión del soluto. Sin embargo debe tenerse presente que este método considera a la solubilidad desde un enfoque fisicoquímico sistemático muy racional, y además, que únicamente haría falta encontrar un método efectivo para el cálculo del parámetro $K$ de Walker, con el cual se calcularía el término $W$ según la expresión $2 K \delta_{1} \delta_{2}$, puesto que los términos $\delta_{1}$ y $\delta_{2}$ serían conocidos, y entonces podría calcularse directamente la solubilidad del fármaco en cualquier composición cosolvente.

\section{CONCLUSIONES}

De todo lo descrito anteriormente se encuentra que la máxima solubilidad de KTP no se obtiene en alguna mezcla cosolvente sino en etanol puro, por lo que el parámetro de solubilidad de este fármaco debe ser inferior al valor correspondiente al del solvente menos polar. La solubilidad experimental del fármaco es menor que la solubilidad ideal en todos los casos, lo que a su vez conduce a coeficientes de actividad mayores que la unidad. Adicionalmente, se ha utilizado satisfactoriamente el MESH en el estudio de la solubilidad de KTP en mezclas $\mathrm{EtOH}+\mathrm{W}$, utilizando para este fin el volumen molar y el parámetro de solubilidad calculados por contribución de grupos. En particular, se ha encontrado una buena capacidad predictiva utilizando un modelo polinómico de quinto orden del parámetro de interacción $W$ en función del parámetro de solubilidad de las mezclas solventes. Sin embargo, al comparar los resultados obtenidos directamente y aquellos obtenidos con el MESH, se encuentra que no hay mejoría significativa en el carácter predictivo al pasar desde el método estrictamente empírico hasta el método extendido de Hildebrand.

\section{AGRADECIMIENTOS}

Agradecemos muy formalmente a la DIB de la Universidad Nacional de Colombia (UNC) por el apoyo financiero y al Departamento de Farmacia de la UNC por facilitar los equipos e instalaciones requeridos para el desarrollo de esta investigación.

\section{REFERENCIAS}

1. Hanson, G. R. En Remington: The Science and Practice of Pharmacy; Gennaro, A.R., ed.; $20^{\text {th }}$ ed.; Lippincott Williams \& Wilkins: Philadelphia, 2000, p. 1456-1460.

2. Jiménez, F.; Martínez, F.; Rev. Colomb. Cienc. Quím. Farm. 1995, 24, 19.

3. Martin, A.; Newburger, J.; Adjei, A.; J. Pharm. Sci. 1980, 69, 659; Martin, A.; Wu, P. L.; J. Pharm. Sci. 1981, 72, 587; Martin, A.; Miralles, M. J.; J. Pharm. Sci. 1982, 71, 439; Martin, A.; Wu, P. L.; Adjei, A.; Mehdizadeh, M.; James, K. C.; Metzler, C.; J. Pharm. Sci. 1982, 71, 1334.

4. Manrique, Y. J.; Pacheco, D. P.; Martínez, F.; Resúmenes del XVII Congreso Farmacéutico Argentino, Villa Carlos Paz, Argentina, 2006; Pacheco, D. P.; Manrique, Y. J.; Vargas, E. F.; Barbosa, H. J.; Martínez, F.; Rev. Colomb. Quím. 2007, 36, 55; Aragón, D. M.; Pacheco, D. P.; Ruidiaz, M. A.; Sosnik, A. D.; Martínez, F.; Vitae Rev. Fac. Quím. Farm. 2008, 15, 113 .

5. Yalkowsky, S. H.; Roseman, T. J.; En Techniques of Solubilization of Drugs; Yalkowsky, S. H., ed.; Marcel Dekker, Inc.: New York, 1981, p. 91-134.

6. Jouyban, A.; Acree Jr, W. E.; J. Pharm. Pharmaceut. Sci. 2006, 9, 262; Jouyban, A.; Pharmazie 2007, 62, 46; Jouyban, A.; Pharmazie 2007, 62,365 .

7. Jouyban, A.; J. Pharm. Pharmaceut. Sci. 2008, 11, 32.

8. Hildebrand, J. H.; Prausnitz, J. M.; Scott, R. L.; Regular and Related Solutions, Van Nostrand Reinhold: New York, 1970.

9. Martin, A.; Bustamante, P.; Anal. Real Acad. Farm. 1989, 55, 175.

10. Martin A.; Bustamante, P.; Chun, A. C. H.; Physical Pharmacy: Physical Chemical Principles in the Pharmaceutical Sciences, $4^{\text {th }}$ ed.; Lea \& Febiger: Philadelphia, 1993, p. 135-137.

11. Hansen, C. M.; Beerbower, A. En Kirk \& Othmer: Encyclopedia of Chemical Technology; Standen, A., ed.; John Wiley \& Sons: New York, 1971, Suppl. 2 ed., p. 889-910.

12. US Pharmacopeia, $28^{\text {th }}$ revision; United States Pharmacopeial Convention, Inc.: Rockville, 2004.

13. Budavari, S.; O’Neil, M. J.; Smith, A.; Heckelman, P. E.; Obenchain Jr., J. R.; Gallipeau, J. A. R.; D’Arecea, M. A. The Merck Index: An 
Encyclopedia of Chemicals, Drugs, and Biologicals, $13^{\text {th }}$ ed. Merck \& Co., Inc.: Whitehouse Station, 2001.

14. Perlovich, G. L.; Kurkov, S. V.; Kinchin, A. N.; Bauer-Brandl, A.; J. Pharm. Sci. 2003, 92, 2502.

15. Jiménez, J.; Manrique, J.; Martínez, F.; Rev. Colomb. Cienc. Quím. Farm. 2004, 33, 145.

16. Barton, A.; Handbook of Solubility Parameters and Other Cohesion Parameters, $2^{\text {nd }}$ ed.; CRC Press: New York, 1991, p. 157-193.

17. Pacheco, D. P.; Martínez, F.; Phys. Chem. Liquids 2007, 45, 581.
18. Manrique, J.; Martínez, F.; Lat. Am. J. Pharm. 2007, 26, 344.

19. Fedors, R. F.; Polym. Eng. Sci. 1974, 14, 147.

20. Yalkowsky, S. H.; Solubility and Solubilization in Aqueous Media, American Chemical Society and Oxford University Press: New York, 1999, p. 180-235.

21. Kimura, F.; Murakami, S.; Fujishiro, R.; J. Solution Chem. 1975, 4, 241.

22. Matsumoto, Y.; Touhara, H.; Nakanishi, K.; Watanabe, N.; J. Chem. Thermodyn. 1977, 9, 801.

23. Rubino, J. T.; Obeng, E. K.; J. Pharm. Sci. 1991, 80, 479. 